\title{
On the Asymptotic Behavior and Radial Symmetry of Positive Solutions of Semilinear Elliptic Equations in $\boldsymbol{R}^{n}$ I. Asymptotic Behavior
}

\author{
YI LI \& WEI-MING NI
}

\section{Introduction}

This paper is motivated by our previous studies on the conformal scalar curvature equation [LN1]

$$
\Delta u+K(x) u^{\frac{n+2}{n-2}}=0
$$

in $\boldsymbol{R}^{n}, n \geqq 3$, where $\Delta=\sum_{i=1}^{n} \frac{\partial^{2}}{\partial x_{i}^{2}}$ is the usual Laplacian and $K$ is a given function, and the Matukuma equation [LN2]

$$
\triangle u+\frac{1}{1+|x|^{2}} u^{p}=0
$$

in $\boldsymbol{R}^{n}, n \geqq 3$, where $p>1$ is a real number. We refer the interested readers to [LN1] and [NY] for the background of (1.1) and (1.2) in Riemannian geometry and astrophysics.

In [LN2] it was established that bounded positive solutions of (1.2) with finite total mass (i.e., $\int_{R^{n}} \frac{u^{p}(x)}{1+|x|^{2}} d x$ ) must be radially symmetric if $p>$ $\frac{n-1}{n-2}$. Our approach there was to first prove that a bounded positive solution of (1.2) with finite total mass must have the fastest possible decay, namely, $O\left(|x|^{2-n}\right)$ near $x=\infty$. Then the method developed in [GNN] applies if $p>\frac{n-1}{n-2}$ since the decay of the term $\frac{1}{1+|x|^{2}} u^{p}(x)$ is faster than $O\left(|x|^{-n-1}\right)$ near $x=\infty$. However, the range $1<p \leqq \frac{n-1}{n-2}$ is left open due to the inad- 
equate decay and new ideas are needed in treating this case. One of the purposes of this paper is to close this gap. (See Theorem 2 below.)

In [LN1] we showed, for instance, that if

$$
K(x)=\frac{1}{1+|x|^{\tau}}
$$

for some $\tau>2$ in $\boldsymbol{R}^{n}$, then (1.1) does not possess any positive solutions tending to zero at $\infty$. On the other hand, it follows from previous works [N], [Na] and [LN1] that every bounded positive solution of (1.1) must tend to a limit $C>0$ at $\infty$, and for every sufficiently small $C>0$, there exists at least one positive radial solution of (1.1) having $C$ as its limit at $\infty$. It thus seems to be a natural question to ask whether all bounded positive solutions of (1.1) with $K$ given by (1.3) are radially symmetric. Again it is the "slow" decay of $K u^{(n+2) /(n-2)}$ that causes the main difficulty here. Attempting to apply the method in [GNN], one immediately encounters the fact that the fundamental tool, Lemma 2.1 in [GNN], no longer holds. In fact, none of the integrals there makes sense when $\tau$ is close to 2. (See our discussions below.) In this paper we shall circumvent this difficulty and give the above question an affirmative answer. (See Theorem 1 below.)

Our key new idea is to obtain precise asymptotic expansions of solutions at $\infty$ which turns out to be sufficient to get the "moving plane" process started near $\infty$. This "moving plane" technique was first devised by A. D. Alexandrov in 1956 and has been used by J. SerRin and many other mathematicians in their work. (See, e.g., [H], [S], and [GNN].) Our paper is close in spirit to [GNN]-both treating semilinear elliptic equations in the entire space $\boldsymbol{R}^{n}$. To make the above discussion slightly more precise and to further illustrate the main ideas involved, we quote one of the results in [GNN]:

Theorem A. ([GNN; p. 380, Theorem 1"]) Let $u$ be a positive $C^{2}$ solution of

$$
\Delta u+g(|x|, u)=0
$$

in $\boldsymbol{R}^{n}, n \geqq 3$, with $u(x)=O\left(|x|^{-m}\right), m>0$, near $\infty$. Assume that

(i) for $r \geqq 0$ and $0<s \leqq u_{0}=\max u, g(r, s)$ is continuous, positive, nondecreasing in $s$ and strictly decreasing in $r$;

(ii) for some $p>(n+1) / m$ and some constant $C>0, g(r, u) \leqq C u^{p}$ for $u \leqq u_{0}$.

Then $u$ is radially symmetric about the origin and $u_{r}<0$ for $r>0$.

To get the "moving plane". process started near $\infty$ in the proof of Theorem $A$, the following lemma was used in a very crucial way in [GNN].

Lemma B. ([GNN; p. 375, Lemma 2.1]) Let $u$ be given by

$$
u(x)=\int_{\boldsymbol{R}^{n}} \frac{f(y)}{|x-y|^{n-2}} d y
$$


with $f(y)=O\left(|y|^{-q}\right)$ at $\infty$ for some $q>n$. Then

$$
\lim _{x \rightarrow \infty}|x|^{n-2} u(x)=\int_{\mathbb{R}^{n}} f(y) d y .
$$

Furthermore if $q>n+1$, then

$$
\lim _{x_{1} \rightarrow \infty} \frac{|x|^{n}}{x_{1}} \frac{\partial u}{\partial x_{1}}(x) \rightarrow-(n-2) \int_{\boldsymbol{R}^{n}} f(y) d y
$$

if $\lambda^{i} \rightarrow \lambda \in \boldsymbol{R}$ and $\left\{x^{i}\right\}$ is a sequence of points going to $\infty$ with $x_{1}^{i}<\lambda^{i}$, then

$$
\frac{\left|x^{i}\right|^{n}}{\lambda^{i}-x_{1}^{i}}\left(u\left(x^{i}\right)-u\left(x^{i \lambda^{i}}\right)\right) \rightarrow 2(n-2) \int_{R^{n}} f(y)\left(\lambda-y_{1}\right) d y
$$

as $i \rightarrow \infty$, where $x=\left(x_{1}, \ldots, x_{n}\right)$, and $x^{\lambda}=\left(2 \lambda-x_{1}, x_{2}, \ldots, x_{n}\right)$ is the reflected point of $x$ with respect to the hyperplane $x_{1}=\lambda$.

Heuristically, the fact that $u(x) \geqq u\left(x^{\lambda}\right)$ for $x$ near $\infty$ with $x_{1}<\lambda$ where $\lambda>0$ is sufficiently large follows from (1.6) and (1.7) since (1.6) already implies that $u$ is strictly decreasing in the $x_{1}$-direction for $x_{1}$ near $\infty$ (if $f \geqq 0$ and $\mathrm{F} 0$ ). This is the starting point of the "moving plane" process. Now, for a bounded positive solution $u$ of (1.1) with $K$ given by (1.3), the decay of the term $K u^{\frac{n+2}{n-2}}$ is precisely $\sim|x|^{-\tau}$ near $x=\infty$, which is not faster than $|x|^{-n-1}$ if $\tau \leqq n+1$, and Lemma $\mathbf{B}$ is not applicable. Similarly, for a bounded positive solution $u$ of (1.2) with finite total mass, the term $\frac{1}{1+|x|^{2}} u^{p}$ has the precise decay $\sim|x|^{-p(n-2)-2}$ near $\infty$, which is again not faster than $|x|^{-n-1}$ if $p \leqq \frac{n-1}{n-2}$. Thus the applicability of Lemma $\mathrm{B}$ is excluded and the question of whether $u$ is radially symmetric is left open.

The crucial new idea in this paper is to obtain the precise asymptotic behavior of solutions near $\infty$ (which enables us to bypass Lemma B). The derivation of such asymptotic expansions of solutions could sometimes be very technical and tedious. However, using them we are able to prove the following results.

Theorem 1. All bounded positive solutions of (1.1) with $K$ given by (1.3) are radially symmetric.

Theorem 2. All bounded positive solutions of (1.2) with finite total mass are radial-. ly symmetric.

As a by-product of our method, we also know precisely how all such solutions behave near $\infty$. For instance, in case of the Matukuma equation we have 
Theorem 3. Let $u$ be a bounded positive solution of (1.2) with finite total mass. Then

$$
\begin{aligned}
u(x)= & \frac{C}{|x|^{n-2}}+\frac{c}{|x|^{n-2+\gamma}}+\cdots+\frac{c}{|x|^{n-2+(2 k+1) \gamma}} \\
& +\frac{c}{|x|^{n-1+\gamma}}+\cdots+\frac{c}{|x|^{n-1+k \gamma}}+O\left(\frac{1}{|x|^{n}}\right)
\end{aligned}
$$

near $x=\infty$, where $\gamma=(p-1)(n-2), k$ is the integer for which $k \gamma \leqq 1<$ $(k+1) \gamma$, and $C>0$ and $c$ are generic constants.

This follows immediately from Theorem 2 and Theorems $2.8,2.12$ and 2.16 in Section 2 below. Our other results in this direction, Theorems 2.41, 2.50 and 2.75, are also of independent interest. Furthermore, our method applies to the more general equation (1.4), and we are able to refine and improve previous results in [GNN] (such as Theorem $A$ quoted above and Theorem 1 in [GNN]); in particular, not only the condition on $p$ is now optimal, the monotonicity assumption of $g(r, u)$ on $u$ is also removed. (See, for instance, Remarks 5.18 and 6.3 of Part II.)

For the convenience of the reader, we group our results into two parts. Part I (containing Sections 1 and 2) is devoted to the derivation of the asymptotic behavior of all possible bounded positive solutions to several classes of general equations including (1.1) and (1.2) where neither the coefficients $K$ nor the solutions $u$ need to be radial. The symmetry results, Theorems 1 and 2, are established in Section 5, Part II, as special cases of much more general results. For the purpose of proving those symmetry results in Section 5, we apply the method developed in Section 2 to general equations including (1.1) and (1.2) under the assumption that $K$ is radial (while still no radial symmetry hypothesis is imposed on solutions). Since we only need the asymptotic expansions of solutions up to the order $|x|^{-(n-1)}$ in Section 5, our asymptotic conditions on $K$ (obtained in Section 2 ) can be considerably relaxed and the proofs are simpler. These are done in Section 3, Part II. (This makes Part II essentially self-contained.) Section 4 contains some very important technical estimates (for example, Lemmas 4.6 and 4.9, which are to replace Lemma $B$ above). Finally, we conclude Part II with a few remarks in Section 6.

\section{Asymptotic Behavior}

In this section we determine the asymptotic behavior of positive solutions of the equation

$$
\Delta u+g(x, u)=0
$$

in $\boldsymbol{R}^{n}, n \geqq 3$. For simplicity we assume that

$$
g(x, u)=K(x) f(u)
$$


where $K \geqq 0$ in $\boldsymbol{R}^{n}, f \geqq 0$ on $\boldsymbol{R}^{+}$, and $K$ and $f$ satisfy some further more precise conditions. In fact, our method is quite general - it applies to more general nonlinearities than (2.2), for instance,

$$
g(x, u)=\Sigma K_{i}(x) f_{i}(u) .
$$

The results in [LN1] guarantee that a bounded positive solution $u$ must tend to a limit $C_{0}$ at $\infty$ if $K(x) f(u)$ decays sufficiently fast near $\infty$. Moreover, Theorem 2.13 in [LN1] gives the second term in the asymptotic expansion of $u$ if $C_{0}$ is positive. In Subsection 2.1 we shall treat the case $C_{0}=0$, and the case $C_{0}>0$ will be further studied in Subsection 2.2. Various extensions and generalizations are included in Subsection 2.3.

Throughout this entire paper we shall use the capital letter $C$ to denote generic positive constants which may vary from line to line and use the lowercase $c$ to denote generic constants (not necessarily positive or negative) which again may vary from line to line. We shall also use the notation $R(x)$ to denote a generic function which is Lipschitz continuous in a neighborhood of the origin 0 with $R(0)=0$.

\subsection{The Zero Limit Case}

Our first step in the zero limit case is the following (also see Theorem 2.32)

Theorem 2.4. Let $u$ be a positive solution of (2.1) with $u(x)=O\left(|x|^{-m}\right)$ near $x=\infty$ for some $m>0$. Suppose that $g(x, u)=K(x) f(u)$ with $K(x)=$ $O\left(|x|^{-\tau}\right)$. near $\infty$ for some $\tau \geqq 0$, that $f(u)=O\left(u^{p}\right)$ near $u=0$ for $u$ positive, and that $\tau+p m>n$. Then $m=n-2$ and

$$
\lim _{x \rightarrow \infty}|x|^{n-2} u(x)=\frac{1}{n(n-2) \omega_{n}} \int_{R^{n}} g(x, u(x)) d x \equiv C_{1}>0
$$

where $\omega_{n}$ is the volume of the unit ball in $\boldsymbol{R}^{n}$. Furthermore,

$$
u(x)-\frac{C_{1}}{|x|^{n-2}}= \begin{cases}O\left(|x|^{-(n-1)}\right) & \text { if } \gamma>1, \\ O\left(|x|^{-(n-1)} \log |x|\right) & \text { if } \gamma=1, \\ O\left(|x|^{-(n-2+\gamma)}\right) & \text { if } \gamma<1,\end{cases}
$$

for $x$ near $\infty$ where $\gamma=\tau+p(n-2)-n$.

Proof. By Lemma 2.3 in [LN1] we have

$$
u(x)=\frac{1}{n(n-2) \omega_{n}} \int_{R^{n}} \frac{g(y, u(y))}{|x-y|^{n-2}} d y .
$$

Thus (2.5) follows from (1.5) in Lemma B (in the Introduction) and consequently $m=n-2$. 
From (2.7) we deduce that

$$
\begin{aligned}
& \left.n(n-2) \omega_{n}|| x\right|^{n-2} u(x)-C_{1} \mid \\
& \leqq \int_{R^{n}}\left|\frac{|x|^{n-2}}{|x-y|^{n-2}}-1\right| g(y, u(y)) d y \\
& =\left(\int_{|y|<\frac{|x|}{2}}+\int_{|x-y|<\frac{|x|}{2}}+\int_{\left[|y|>\frac{|x|}{2}\right] \cap\left[|x-y|>\frac{|x|}{2}\right]}\right)\left|\frac{|x|^{n-2}}{|x-y|^{n-2}}-1\right| g(y, u(y)) d y \\
& \equiv I_{1}+I_{2}+I_{3}
\end{aligned}
$$

where $I_{1}, I_{2}$ and $I_{3}$ are defined by the last equality. We now estimate $I_{1}, I_{2}$ and $I_{3}$ separately. In the region $\left\{y \in \boldsymbol{R}^{n}|| y \mid<\frac{|x|}{2}\right\}$ the inequality $\frac{|x|}{2}<|x-y|<$ $\frac{3|x|}{2}$ holds. Thus

$$
\begin{aligned}
I_{1} & =\int_{|y|<\frac{|x|}{2}}\left|\frac{|x|^{n-2}-|x-y|^{n-2}}{|x-y|^{n-2}}\right| g(y, u(y)) d y \\
& =\int_{|y|<\frac{|x|}{2}}\left(\frac{|x|^{n-3}+\cdots+|x-y|^{n-3}}{|x-y|^{n-2}}\right) \frac{\left.|| x\right|^{2}-|x-y|^{2} \mid}{|x|+|x-y|} g(y, u(y)) d y \\
& \leqq \frac{C}{|x|} \int_{|y|<\frac{|x|}{2}}^{|y| g(y, u(y)) d y} \\
& \leqq \frac{C}{|x|} \int_{|y|<\frac{|x|}{2}} \frac{(1+|y|)^{\tau+p(n-2)}}{(1+|y|} y
\end{aligned}
$$

since $\left.|| x\right|^{2}-|x-y|^{2}|=| x \cdot x-(x-y) \cdot(x-y)|\leqq| y \mid(|y|+2|x|)$. Noting that $\tau+p(n-2)=\gamma+n$ we have

$$
I_{1} \leqq \frac{C}{|x|} \int_{0}^{\frac{|x|}{2}} \frac{r^{n}}{(1+r)^{\gamma+n}} d r \leqq \begin{cases}C|x|^{-1} & \text { if } \gamma>1 \\ C|x|^{-1} \log |x| & \text { if } \gamma=1 \\ C|x|^{-\gamma} & \text { if } \gamma<1\end{cases}
$$


To estimate $I_{2}$ we observe that in the region $\left\{y \in \boldsymbol{R}^{n}|| x-y \mid<\frac{|x|}{2}\right\}$ we have $\frac{|x|}{2}<|y|<\frac{3|x|}{2}$. Thus, setting $r=|x-y|$ we obtain from the definition of $I_{2}$ that

$$
I_{2} \leqq C \int_{0}^{\frac{|x|}{2}}\left(|x|^{n-2} r^{2-n}+1\right) \frac{1}{|x|^{\gamma+n}} r^{n-1} d r \leqq \frac{C}{|x|^{\gamma}} .
$$

Finally it is easy to see that if $|x-y|>\frac{|x|}{2}$, then

and therefore

$$
\left|\frac{|x|^{n-2}}{|x-y|^{n-2}}-1\right| \leqq C
$$

$$
I_{3} \leqq C \int_{|y| \geqq \frac{|x|}{2}} \frac{1}{|y|^{\gamma+n}} d y=\frac{C}{|x|^{\gamma}} .
$$

Combining the estimates for $I_{1}, I_{2}$ and $I_{3}$ we obtain (2.6). Q.E.D.

We now come to the main results of this subsection, which refine Theorem 2.4 by giving higher-order terms in the asymptotic expansion (2.6) of $u$. It seems natural that if we need more precise asymptotic behavior of solutions, we have to have more precise assumptions on the nonlinearity. Although our method handles very general nonlinearities, results in such cases could be very complicated. Thus, to make the method transparent we only include the following theorems as examples to illustrate the ideas involved. (See Remark 2.30 for more complicated nonlinearities.)

Theorem 2.8. Suppose that the assumptions of Theorem 2.4 hold and that $\gamma>1$. Suppose further that

$$
K(x)=|x|^{-\tau}\left(C+O\left(|x|^{-\mu}\right)\right)
$$

near $x=\infty$ for some $\mu>2-\gamma$, and that for some $q$ with $q(n-2)>2-\gamma$

$$
f(u)=u^{p}\left(C+O\left(u^{q}\right)\right)
$$

near $u=0$ for $u>0$. Then

$$
u(x)=\frac{C}{|x|^{n-2}}+\frac{a \cdot x}{|x|^{n}}+\frac{c}{|x|^{n-2+\gamma}}+R\left(\frac{x}{|x|^{2}}\right) \frac{1}{|x|^{n-1}}
$$

near $x=\infty$ where $a \in \boldsymbol{R}^{n}$ is a constant vector.

Theorem 2.12. Suppose that the assumptions of Theorem 2.4 hold and that $\gamma=1$. Suppose further that

$$
K(x)=|x|^{-\tau}\left(C+c|x|^{-1}+O\left(|x|^{-\mu}\right)\right)
$$


near $x=\infty$ for some $\mu>2-\gamma(=1)$, and that for some $q$ with $q(n-2)>$ $2-\gamma(=1)$

$$
f(u)= \begin{cases}u^{p}\left(C+O\left(u^{q}\right)\right) & \text { if } n>3 \\ u^{p}\left(C+c u+O\left(u^{q}\right)\right) & \text { if } n=3\end{cases}
$$

near $u=0$ for $u>0$. Then

$$
u(x)=\frac{C}{|x|^{n-2}}+\frac{c}{|x|^{n-1}}+\frac{a \cdot x}{|x|^{n}}+R\left(\frac{x}{|x|^{2}}\right) \frac{1}{|x|^{n-1}}
$$

near $x=\infty$, where $a \in \boldsymbol{R}^{n}$ is a constant vector.

Theorem 2.16. Suppose that the assumptions of Theorem 2.4 hold and that $\gamma<1$. Suppose further that (2.13) and (2.14) hold. Then

$$
\begin{aligned}
& u(x)=\frac{C}{|x|^{n-2}}+\frac{c}{|x|^{n-2+\gamma}}+\cdots+\frac{c}{|x|^{n-2+(2 k+1) \gamma}}+\frac{c}{|x|^{n-1+\gamma}}+\cdots+\frac{c}{|x|^{n-1+k \gamma}} \\
& +\frac{a \cdot x}{|x|^{n}}\left(1+\frac{c}{|x|^{\nu}}+\cdots+\frac{c}{|x|^{k \gamma}}\right)+R\left(\frac{x}{|x|^{2}}\right) \frac{1}{|x|^{n-1}}
\end{aligned}
$$

near $x=\infty$, where $k$ is the integer such that $k \gamma \leqq 1<(k+1) \gamma$ and $a \in \boldsymbol{R}^{n}$ is a constant vector.

The proof of Theorem 2.16 is far more complicated than that of Theorems 2.8 and 2.12. We shall therefore give a detailed proof of Theorem 2.16 and merely sketch the proofs of Theorems 2.8 and 2.12 .

Proof of Theorem 2.16. The proof is divided into two steps.

Step I. First observe that if $n \geqq 4$, we may then choose $0<q<1$ such that $q(n-2)>2-\gamma$. Thus in this case we may rewrite

$$
\begin{aligned}
f(u) & =u^{p}\left(C+c u-c u+O\left(u^{q}\right)\right) \\
& =u^{p}\left(C+c u+O\left(u^{q}\right)\right)
\end{aligned}
$$

near $u=0$ for $u<0$. Thus there is no need to consider the cases $n \geqq 4$ and $n=3$ separately.

We shall now use the Kelvin transform to convert the problem to a neighborhood of the origin, which is technically easier to handle. Setting

$$
y=\frac{x}{|x|^{2}}, \quad v(x)=|x|^{2-n} u(y)=|y|^{n-2} u(y)=C_{1}+v_{1}(x)
$$

where $v_{1}(x)=O\left(|x|^{\gamma}\right)$ near $x=0$ by (2.6), we obtain, by standard computation, for $x$ near the origin, say, in $B_{1}(0) \backslash\{0\}$, that

$$
0=\Delta v+|x|^{\gamma-2} v^{p}\left[C+c|x|+O\left(|x|^{\mu}\right)\right]\left[C+c|x|^{n-2} v+O\left(\left(|x|^{n-2} v\right)^{q}\right)\right] .
$$


Thus using (2.19) we have

$$
\begin{aligned}
0= & \Delta v+|x|^{\gamma-2}\left[C_{1}+O\left(|x|^{\gamma}\right)\right]^{p} \\
& \times\left[C+c|x|+O\left(|x|^{2}\right)+O\left(|x|^{\mu}\right)+O\left(|x|^{(n-2) q}\right)+O\left(|x|^{n-2+\gamma}\right)\right] \\
= & \Delta v+|x|^{\gamma-2}\left[C_{1}^{p}+O\left(|x|^{\gamma}\right)\right] \\
& \times\left[C+c|x|+O\left(|x|^{2}\right)+O\left(|x|^{\mu}\right)+O\left(|x|^{(n-2) q}\right)+O\left(|x|^{n-2+\gamma}\right)\right] \\
= & \Delta v+|x|^{\gamma-2}\left[C+O\left(|x|^{\gamma}\right)+O\left(|x|^{\mu}\right)+O\left(|x|^{2}\right)+O\left(|x|^{(n-2) q}\right)\right] \\
= & \Delta v+C|x|^{\gamma-2}+O\left(|x|^{2 \gamma-2}\right) \\
\equiv & \Delta v+\tilde{C}|x|^{\gamma-2}+f_{1}(x)
\end{aligned}
$$

since $2 \gamma-2<0, \gamma-2+\mu>0$ and $q(n-2)+\gamma-2>0$, where $f_{1}$ and $\tilde{C}>0$ are defined by the last equality. Setting

$$
w(x)=C_{1}-\frac{\tilde{C}}{\gamma(\gamma+n-2)}|x|^{\gamma}+F_{1}(x), \quad 0<|x|<1
$$

where $F_{1}$ is the Newtonian potential of $f_{1}$, we see that $\Delta(v-w)=0$ in $B_{1}(0) \backslash\{0\}$. Since $f_{1} \in L^{p}\left(B_{1}(0)\right)$ for $1<p<\frac{n}{2-2 \gamma}$, standard elliptic estimates imply that $F_{1} \in W^{2, p}\left(B_{1}(0)\right)$ and thus $F_{1}$ is Hölder continuous near $x=0$ by the Sobolev Embedding Theorem and the fact that $\frac{n}{2-2 \gamma}>\frac{n}{2}$. This in particular guarantees that $v-w$ is bounded and therefore $x=0$ is a removable singularity of the harmonic function $v-w$, i.e., $v \equiv w+H$ in $B_{1}(0)$ where $H$ is a harmonic function in the entire ball $B_{1}(0)$. If $2 \gamma>1$, then $\frac{n}{2-2 \gamma}>n$ and $F_{1} \in C^{1, \delta}\left(B_{1}(0)\right)$ for any $\delta<2 \gamma-1$ by the Sobolev Embedding Theorem. Thus if we set $a=\nabla\left(F_{1}+H\right)(0)$, then

$$
v(x)=C_{1}-\frac{\tilde{C}}{\gamma(\gamma+n-2)}|x|^{\gamma}+a \cdot x+v_{2}(x)
$$

near $x=0$ where $v_{2}(x)=O\left(|x|^{1+\delta}\right)$ at $x=0$ for all $\delta<2 \gamma-1$.

If $2 \gamma \leqq 1$, then again by standard elliptic regularity estimates we have

$$
v(x)=C_{1}-\frac{\tilde{C}}{\gamma(\gamma+n-2)}|x|^{\gamma}+v_{2}(x)
$$

where $v_{2}(x)=O\left(|x|^{\delta}\right)$ near $x=0$ for all $\delta<2 \gamma$. Now we proceed inductively. Assume that for $j \leqq k-1$ we have

$$
v(x)=C_{1}+c|x|^{\gamma}+\cdots+c|x|^{(j-1) \gamma}+v_{j}(x)
$$


where $v_{j}(x)=O\left(|x|^{\delta}\right)$ for all $\delta<j y$ near $x=0$. Substituting (2.23) into (2.20) we obtain, after a similar computation as above, that

$$
\begin{aligned}
0= & \Delta v+|x|^{\gamma-2}\left[C^{p}+c|x|^{\gamma}+\cdots+c|x|^{(j-1) \gamma}+O\left(|x|^{\delta}\right)\right] \\
& \times\left[C+c|x|+O\left(|x|^{2}\right)+O\left(|x|^{\mu}\right)+O\left(|x|^{q(n-2)}\right)+O\left(|x|^{n-2+\gamma}\right)\right] \\
= & \Delta v+C|x|^{\gamma-2}+c|x|^{2 \gamma-2}+\cdots+c|x|^{j \gamma-2}+O\left(|x|^{\delta+\gamma-2}\right)+O\left(|x|^{\gamma-1}\right) .
\end{aligned}
$$

(Here, again we have used the assumptions that $\mu+\gamma-2>0$ and $q(n-2)+\gamma-2>0$.) Since $(j+1) \gamma \leqq k \gamma \leqq 1$ and $\gamma-1>\delta+\gamma-2$, it follows that

$$
0=\Delta v+C|x|^{\gamma-2}+c|x|^{2 \gamma-2}+\cdots+c|x|^{j \gamma-2}+O\left(|x|^{\delta+\gamma-2}\right)
$$

near $x=0$ for all $\delta<j \gamma$. Now the arguments leading to (2.22) may be repeated here to yield that

$$
v(x)=C_{1}+c|x|^{\gamma}+\cdots+c|x|^{j \gamma}+v_{j+1}(x)
$$

near $x=0$ where $v_{j+1}(x)=O\left(|x|^{\delta}\right)$ near $x=0$ for all $\delta<(j+1) \gamma$. Since this holds for all $j \leqq k-1$ we conclude that

$$
v(x)=C_{1}+c|x|^{\gamma}+\cdots+c|x|^{(k-1) \gamma}+v_{k}(x)
$$

near $x=0$ where $v_{k}(x)=O\left(|x|^{\delta}\right)$ at $x=0$ for all $\delta<k \gamma$. Iterating the above argument by substituting (2.24) into $(2.20)$ we obtain

$$
0=\Delta v+C|x|^{\gamma-2}+c|x|^{2 \gamma-2}+\cdots+c|x|^{k \gamma-2}+O\left(|x|^{\delta+\gamma-2}\right)
$$

near $x=0$ for all $\delta<k \gamma$. Note that since $(k+1) \gamma>1, \delta+\gamma-2>-1$ for $\delta$ sufficiently close to $k \gamma$, it follows from the arguments leading to (2.21) that

$$
v(x)=C_{1}+c|x|^{\gamma}+\cdots+c|x|^{k \gamma}+a \cdot x+v_{k+1}(x)
$$

near $x=0$ where $v_{k+1}(x)=O\left(|x|^{1+\delta}\right)$ near $x=0$ for all $\delta<(k+1) \gamma-1$ and $a$ is a constant vector in $\boldsymbol{R}^{n}$.

Step II. Iterating the arguments in Step I, we substitute (2.25) into (2.20) and obtain after a tedious computation that

$$
\begin{aligned}
0= & \Delta v+\left[C|x|^{\gamma-2}+c|x|^{2 \gamma-2}+\cdots+c|x|^{(k+1) \gamma-2}+p(a \cdot x)|x|^{\gamma-2}\right. \\
& \left.+O\left(|x|^{\gamma-1+\delta}\right)+c|x|^{\gamma-1}+c|x|^{2 \gamma-1}+\cdots+c|x|^{k \gamma-1}\right]
\end{aligned}
$$

near $x=0$ for all $\delta<(k+1) \gamma-1$. Similarly we conclude (as we did in (2.21)) that near $x=0$,

$$
\begin{aligned}
v(x)= & C_{1}+c|x|^{\gamma}+\cdots+c|x|^{(k+1) \gamma}+(a \cdot x)\left(1+c|x|^{\gamma}\right) \\
& +c|x|^{\gamma+1}+c|x|^{2 \gamma+1}+\cdots+c|x|^{k \gamma+1}+O\left(|x|^{\gamma+1+\delta}\right)
\end{aligned}
$$

where $a \in \boldsymbol{R}^{n}$ is a constant vector; in fact, $a=\nabla\left[v(x)-\left(C_{1}+c|x|^{\gamma}+\cdots\right.\right.$ $\left.\left.+c|x|^{k \gamma}\right)\right]\left.\right|_{x=0}$. (Note that in deriving (2.26) we have used the fact that $\Delta\left((a \cdot x)|x|^{\gamma}\right)=c(a \cdot x)|x|^{\gamma-2}, x \neq 0$.) If $\gamma+1+\delta \geqq 2$ for some $\delta<(k+1) \gamma-1$, 
then $(\gamma+1)+(k+1) \gamma-1>2$, which implies that $k=1$, and we stop at (2.26). Otherwise, since $\gamma+1+(k+1) \gamma-1 \leqq 2 \gamma+1$ we may simplify (2.26) as

$$
\begin{aligned}
v(x)= & C_{1}+c|x|^{\gamma}+\cdots+c|x|^{(k+1) \gamma}+(a \cdot x)\left(1+c|x|^{\gamma}\right) \\
& +c|x|^{\gamma+1}+O\left(|x|^{\gamma+1+\delta}\right)
\end{aligned}
$$

near $x=0$ for all $\delta \leqq(k+1) \gamma-1(\leqq \gamma)$. Repeating this process, we finally arrive at

$$
\begin{aligned}
v(x)= & C_{1}+c|x|^{\gamma}+\cdots+c|x|^{2 k \gamma}+(a \cdot x)\left(1+c|x|^{\gamma}+\cdots+c|x|^{k \gamma}\right) \\
& +c|x|^{\gamma+1}+\cdots+c|x|^{k \gamma+1}+v_{2 k+1}(x)
\end{aligned}
$$

where $v_{2 k+1}(0)=0, \nabla v_{2 k+1}(0)=0$ and $v_{2 k+1} \in C^{k \gamma+1+\delta}$ near $x=0$ for all $\delta<(k+1) \gamma-1$. Since $k \gamma+1+\delta<k \gamma+1+(k+1) \gamma-1=(2 k+1) \gamma$, which may still be less than or equal to 2 , we need to iterate the above argument once more to obtain

$$
\begin{aligned}
v(x)= & C_{1}+c|x|^{\gamma}+\cdots+c|x|^{(2 k+1) \gamma}+(a \cdot x)\left(1+c|x|^{\gamma}+\cdots+c|x|^{k \gamma}+c|x|^{(k+1) \gamma}\right) \\
& +c|x|^{\gamma+1}+\cdots+c|x|^{k \gamma+1}+c|x|^{(k+1) \gamma+1}+v_{2 k+2}(x)
\end{aligned}
$$

near $x=0$ where $v_{2 k+2} \in C^{(k+1) \gamma+1+\delta}\left(B_{1}(0)\right)$ with $v_{2 k+2}(0)=0, \nabla v_{2 k+2}(0)=0$ for all $\delta<(k+1) \gamma-1$. Since $(k+1) \gamma+1>2$, we combine the three terms $(a \cdot x)|x|^{(k+1) \gamma},|x|^{(k+1) \gamma+1}$ and $v_{2 k+2}$ into a single $C^{2}$ function $\varphi$ in $B_{1}(0)$. It is then easy to see that the function $R(x)$ defined by

$$
R(x)= \begin{cases}\varphi(x) /|x|, & x \neq 0, \\ 0, & x \neq 0\end{cases}
$$

is Lipschitz continuous in $B_{1}(0)$. Thus it follows that, near $x=0$,

$$
\begin{aligned}
v(x)= & C_{1}+c|x|^{\gamma}+\cdots+c|x|^{(2 k+1) \gamma}+(a \cdot x)\left(1+c|x|^{\gamma}+\cdots+c|x|^{k \gamma}\right) \\
& +c|x|^{\gamma+1}+\cdots+c|x|^{k \gamma+1}+R(x)|x|
\end{aligned}
$$

and (2.17) follows from (2.28) and (2.19). Q.E.D.

We now come to the

Proof of Theorem 2.12. We proceed as in the proof of Theorem 2.16. Following the argument leading to $(2.20)$ we now have instead

$$
0=\Delta v+|x|^{-1} v^{p}\left[C+c|x|+O\left(|x|^{1+\delta}\right)+O\left(|x|^{\mu}\right)+O\left(|x|^{(n-2) q}\right)\right]
$$

near $x=0$ for any $\delta<1$, where $v(x)=C_{1}+v_{1}(x)$ given by (2.19) and

$$
v_{1}(x)=O(-|x| \log |x|)=O\left(|x|^{\delta}\right)
$$

near $x=0$ for any $\delta<1$, guaranteed by (2.6). From (2.29) it follows that

$$
0=\Delta v+C|x|^{-1}+O\left(|x|^{-1+\delta}\right)
$$


near $x=0$ for any $\delta<1$, since $\mu>1$ and $q(n-2)>1$. Thus

$$
v(x)=C_{1}+c|x|+a \cdot x+O\left(|x|^{1+\delta}\right)
$$

near $x=0$ for any $\delta<1$ where $a \in R^{n}$ is a constant vector. Substituting this back into (2.29) we obtain that

$0=\Delta v+C|x|^{-1}+c+p(a \cdot x)|x|^{-1}+O\left(|x|^{\delta}\right)+O\left(|x|^{\mu-1}\right)+O\left(|x|^{(n-2) q-1}\right)$

near $x=0$, for all $\delta<1$. This again leads to

$$
v(x)=C_{1}+c|x|+(a \cdot x)(1+c|x|)+v_{2}(x)
$$

near $x=0$, where $v_{2}(0)=0, \nabla v_{2}(0)=0$ and $v_{2} \in C^{2, \delta^{\prime}}\left(B_{1}(0)\right)$ for all $\delta^{\prime}<\min \{1, \mu-1,(n-2) q-1\}$. This can clearly be rewritten as

$$
v(x)=C_{1}+c|x|+a \cdot x+R(x)|x|
$$

near $x=0$, where $R(x)$ is a Lipschitz continuous function in $B_{1}(0)$ with $R(0)=0$. Transforming back via (2.19), we have (2.15). Q.E.D.

Finally we include a brief argument for Theorem 2.8. (Observe that both $\mu$ and $q$ may be chosen to be less than 1 in this case as long as $n \geqq 3$.)

Proof of Theorem 2.8. We proceed as in the proof of Theorem 2.16. Following the argument leading to $(2.20)$, we arrive at

$$
0=\Delta v+|x|^{\gamma-2} v^{p}\left[C+c|x|+O\left(|x|^{\mu}\right)+O\left(|x|^{q(n-2)}\right)\right]
$$

near $x=0$ where $v(x)=C_{1}+v_{1}(x)$ given by (2.19) and $v_{1}(x)=O(|x|)$ near $x=0$. Therefore

$$
0=\Delta v+C|x|^{\gamma-2}+O\left(|x|^{\gamma-1}\right)+O\left(|x|^{\mu+\gamma-2}\right)+O\left(|x|^{q(n-2)+\gamma-2}\right)
$$

near $x=0$. Since $\gamma-1>0, \mu+\gamma-2>0$, and $q(n-2)+\gamma-2>0$, it follows that near $x=0$

$$
v(x)=C_{1}+c|x|^{\gamma}+a \cdot x+v_{2}(x)
$$

where $v_{2} \in C^{2}\left(B_{1}(0)\right)$ with $v_{2}(0)=0$ and $\nabla v_{2}(0)=0$. Again we may write $v_{2}(x)=R(x)|x|$ in $B_{1}(0)$ and then (2.11) follows immediately from (2.19). Q.E.D.

Remark 2.30. For a nonlinearity of the following form

$$
g(x, u)=\frac{1}{1+|x|^{\tau_{1}}} u^{p_{1}}+\frac{1}{1+|x|^{\tau_{2}}} u^{p_{2}}
$$

Theorems 2.8, 2.12 and 2.16 may not apply. However, the method does apply. In fact, the techniques used in the proofs above apply to nonlinearities of the form (2.3) with $K_{i}, f_{i}$ satisfying the hypotheses of Theorems $2.8,2.12$ or 2.16. In this more general case the asymptotic expansions of solutions $u$ are similar to (2.11), (2.15) and (2.17) except now terms with exponents involving various 
combinations of $\gamma_{i}=\tau_{i}+p_{i}(n-2)-n$ appear and make the asymptotic expansions of $u$ look far more complicated than (2.11), (2.15) or (2.17). We shall leave the details to the interested readers.

\subsection{The Positive Limit Case}

For simplicity, in this subsection we restrict our consideration to the equation

$$
\Delta u+K(x) u^{p}=0
$$

in $\boldsymbol{R}^{n}, n \geqq 3$, where $K \geqq 0$ in $\boldsymbol{R}^{n}$ and $p>1$. First, we summarize the known results:

Theorem 2.32. Let $u$ be a bounded positive solution of (2.31) and let $K \geqq 0$ in $\boldsymbol{R}^{n}$ with $K(x)=O\left(|x|^{-\tau}\right)$ near $x=\infty$ for some $\tau>2$. Then $C_{0}=\lim _{x \rightarrow \infty} u(x)$ always exists. Moreover, if $C_{0}=0$, then $u(x)=O\left(|x|^{2-n}\right)$ near $\infty$; if $C_{0}>0$ and $K \sim|x|^{-\tau}$ near $\infty$, then

$$
\left(u(x)-C_{0}\right) \sim \begin{cases}C|x|^{2-n} & \text { if } \tau>n, \\ C|x|^{2-n} \log |x| & \text { if } \tau=n, \\ C|x|^{2-\tau} & \text { if } n>\tau>2 .\end{cases}
$$

(Here we use notation " $f \sim g$ near $\infty$ " to denote that there exist two positive constants $C_{1}, C_{2}$ such that $C_{1} f(x) \geqq g(x) \geqq C_{2} f(x)$ for all $x$ near $\infty$.) This theorem follows from Theorems 2.9 and 2.13 in [LN1]. The rest of this subsection will be devoted entirely to a refinement of Theorem 2.32 for the case $C_{0}>0$ (while the corresponding result for the case $C_{0}=0$ is already contained in Subsection 2.1).

First, we take up the case that $\tau>n$. As in Section 2 of [LN1], we have

$$
u(x)=C_{0}+\frac{1}{n(n-2) \omega_{n}} \int_{\mathbb{R}^{n}} \frac{K(y) u^{p}(y)}{|x-y|^{n-2}} d y \equiv C_{0}+u_{1}(x)
$$

where $u_{1}$ is defined by the last equality. It follows immediately from the proof of Theorem 2.4 that if $K(x)=O\left(|x|^{-\tau}\right)$ near $\infty$, then there exists $C_{1}>0$ such that

$$
u_{1}(x)-\frac{C_{1}}{|x|^{n-2}}= \begin{cases}O\left(|x|^{1-n}\right) & \text { if } \gamma>1, \\ O\left(|x|^{1-n} \log |x|\right) & \text { if } \gamma=1, \\ O\left(|x|^{2-n-\gamma}\right) & \text { if } \gamma<1\end{cases}
$$

near $x=\infty$, where $y=\tau-n$ (in this subsection). The case that $\gamma>1$ is the easiest and will be considered first. To refine Theorem 2.32 in this case, we need to assume further that there exists $\mu>\max \{2-\gamma, 0\}$ such that

$$
K(x)=\frac{1}{|x|^{\tau}}\left(C+o\left(\frac{1}{|x|^{\mu}}\right)\right)
$$


near $x=\infty$. Using the Kelvin transform, we set

$$
y=\frac{x}{|x|^{2}}, \quad v(x)=|x|^{2-n} u_{1}(y)=|y|^{n-2} u_{1}(y) \equiv C_{1}+v_{1}(x)
$$

where $v_{1}$ is defined by the last equality and $v_{1}(x)=O(|x|)$ near $x=0$ by (2.35). From the equation (2.31) we derive that

$$
\begin{aligned}
0 & =\Delta v+|x|^{\gamma-2}\left[C+O\left(|x|^{\mu}\right)\right]\left[C_{0}+|x|^{n-2}\left(C_{1}+v_{1}(x)\right)\right]^{p} \\
& =\Delta v+C|x|^{\gamma-2}+C|x|^{\gamma+n-4}+O\left(|x|^{\delta}\right)
\end{aligned}
$$

near $x=0$, for $0<\delta<\min \{\gamma, \gamma+\mu-2\}$. Since $\delta>0$, similar but simpler arguments used in deriving (2.21) imply that

$$
v(x)=C_{1}+c|x|^{\gamma}+c|x|^{\gamma+n-2}+a \cdot x+v_{2}(x)
$$

near $x=0$, where $a$ is a constant vector in $\boldsymbol{R}^{n}$ and $v_{2} \in C^{2}\left(B_{1}(0)\right)$ with $v_{2}(0)=0, \nabla v_{2}(0)=0$. Since $\gamma>1$, we can combine $v_{2}$ and $|x|^{\gamma+n-2}$ into a term of the form $R(x)|x|$ where $R$ is Lipschitz continuous in $B_{1}(0)$ with $R(0)=0$.

Next we consider the case $\gamma \leqq 1$. In this case we assume similarly that there exists $\mu>2-\gamma$ such that

$$
K(x)=\frac{1}{|x|^{\tau}}\left(C+\frac{c}{|x|}+O\left(\frac{1}{|x|^{\mu}}\right)\right)
$$

near $\infty$. We may assume without loss of generality that $\mu<2$.

If $\gamma<1$, it follows from (2.35) that $|x|^{n-2} u_{1}(x)-C_{1}=O\left(|x|^{-\gamma}\right)$ near $\infty$. Thus after the change of variables (2.36) where now $v_{1}(x)=O\left(|x|^{\gamma}\right)$ near $x=0$, the equation (2.31) becomes

$$
\text { (2.38) } \begin{aligned}
0 & =\Delta v+|x|^{\gamma-2}\left[C+c|x|+O\left(|x|^{\mu}\right)\right]\left[C_{0}+|x|^{n-2}\left(C_{1}+v_{1}(x)\right)\right]^{p} \\
& =\Delta v+C|x|^{\gamma-2}+c|x|^{\gamma-1}+C|x|^{\gamma+n-4}+O\left(|x|^{n+2 \gamma-4}\right)+O\left(|x|^{\gamma+\mu-2}\right)
\end{aligned}
$$

near $x=0$. Since $n+2 \gamma-4 \geqq 2 \gamma-1>-1$ and $\gamma+\mu-2>0$, the same arguments leading to (2.21) give that

$$
\begin{aligned}
v(x) & =C_{1}+c|x|^{\gamma}+c|x|^{\gamma+1}+c|x|^{\gamma+n-2}+a \cdot x+\tilde{v}_{2}(x) \\
& \equiv C_{1}+c|x|^{\gamma}+a \cdot x+v_{2}(x)
\end{aligned}
$$

where $a$ is a constant vector, $\tilde{v}_{2}$ and $v_{2}$ are $C^{1, \delta}\left(B_{1}(0)\right)$ functions for $0<\delta<\gamma$, and $v_{2}(0)=\tilde{v}_{2}(0)=0, \nabla v_{2}(0)=\nabla \tilde{v}_{2}(0)=0$. Substituting this back into (2.38) we have

$$
0=\Delta v+C|x|^{\gamma-2}+c|x|^{\gamma-1}+c|x|^{\gamma+n-4}+c|x|^{2 \gamma+n-4}+O\left(|x|^{\delta}\right)
$$

near $x=0$. Similarly, we conclude as before that

$$
v(x)=C_{1}+c|x|^{\gamma}+c|x|^{\gamma+1}+c|x|^{\gamma+n-2}+c|x|^{2 \gamma+n-2}+a \cdot x+R(x)|x|
$$

near $x=0$. 
The case that $\gamma=1$ may be handled in a very similar fashion. Recall from (2.35) and (2.36) that $|x|^{n-2} u_{1}^{\prime}(x)-C_{1}=O\left(|x|^{-\delta}\right.$ ) near $x=\infty$ for any $\delta<1$. Thus, after the application of the Kelvin transform (2.36) equation (2.31) becomes

$$
0=\Delta v+C|x|^{-1}+c+O\left(|x|^{\mu+\delta-2}\right)
$$

near $x=0$, for any $\delta<1$, and it follows that

$$
v(x)=C_{1}+c|x|+a \cdot x+R(x)|x|
$$

near $x=0$.

The above discussion may be summarized as follows.

Theorem 2.41. Let $u$ be a bounded positive solution of (2.31). Suppose that $K \geqq 0$ in $\boldsymbol{R}^{n}$ with $K(x)=O\left(|x|^{-\tau}\right)$ near $x=\infty$ for some $\tau>n$, and that $\gamma=\tau-n$. Then $C_{0}=\lim _{x \rightarrow \infty} u(x)$ always exists. If $C_{0}>0$, then the following statements hold:

(i) If $\tau>n+1$ and

$$
K(x)=\frac{1}{|x|^{\tau}}\left(C+o\left(\frac{1}{|x|^{\mu}}\right)\right)
$$

near $x=\infty$ for some $\mu>\max \{0,2-\gamma\}$, then

$$
u(x)=C_{0}+\frac{C_{1}}{|x|^{n-2}}+\frac{a \cdot x}{|x|^{n}}+\frac{c}{|x|^{n-2+\gamma}}+R\left(\frac{x}{|x|^{2}}\right) \frac{1}{|x|^{n-1}}
$$

near $x=\infty$, where $R$ is Lipschitz continuous in $B_{1}(0)$ with $R(0)=0$.

(ii) If $\tau \leqq n+1$ and

$$
K(x)=\frac{1}{|x|^{\tau}}\left(C+\frac{c}{|x|}+O\left(\frac{1}{|x|^{\mu}}\right)\right)
$$

near $x=\infty$ for some $\mu>2-\gamma$, then

$$
u(x)=C_{0}+\frac{C_{1}}{|x|^{n-2}}+\frac{c}{|x|^{n-1}}+\frac{a \cdot x}{|x|^{n}}+R\left(\frac{x}{|x|^{2}}\right) \frac{1}{|x|^{n-1}}
$$

near $x=\infty$ when $\tau=n+1$,

$$
\begin{aligned}
u(x)= & C_{0}+\frac{C_{1}}{|x|^{n-2}}+\frac{c}{|x|^{n-2+\gamma}}+\frac{c}{|x|^{n-1+\gamma}}+\frac{c}{|x|^{2 n-4+\gamma}} \\
& +\frac{c}{|x|^{2 n-4+2 y}}+\frac{a \cdot x}{|x|^{n}}+R\left(\frac{x}{|x|^{2}}\right) \frac{1}{|x|^{n-1}}
\end{aligned}
$$

near $x=\infty$ when $\tau<n+1$.

This theorem follows immediately from (2.37), (2.39), (2.40) and the Kelvin transform (2:36). 
Remark 2.45. Combining Theorems 2.32, 2.8 and 2.41 we see that if $K(x)=$ $|x|^{-\tau}\left(C+c|x|^{-1}+O\left(|x|^{-\mu}\right)\right)$ near $\infty$ for some $\tau>n$ and $\mu>2+n-\tau$, then the asymptotic behavior of all bounded positive solutions of (2.31) is well understood. This fact will enable us to prove that in this case all bounded positive solutions of (2.31) are radially symmetric if $K$ is radially symmetric and decreasing in $|x|$. (See $\S 5$ in Part II.)

Next we consider the case $\tau=n$. This case needs a little more care. Let $u$ be a bounded positive solution of (2.31). Then again we may write $u=C_{0}+u_{1}$ where $u_{1}$ is defined in (2.34). By Lemma 2.3 in [LN1] we have $0 \leqq u_{1}(x) \leqq C|x|^{2-n} \log |x|$ near $x=\infty$. Assume that for some $\mu>2$

$$
K(x)=|x|^{-n}\left(\tilde{C}+c|x|^{-1}+c|x|^{-2}+O\left(|x|^{-\mu}\right)\right)
$$

near $x=\infty$. Then we have from (2.31) that near $x=\infty$

$$
0=\Delta u_{1}+\tilde{C} C_{0}^{p}|x|^{-n}+O\left(|x|^{-n-1}\right)+O\left(|x|^{2-2 n} \log |x|\right) .
$$

Setting

near $x=\infty$, we have

$$
u_{2}(x)=u_{1}-\frac{\tilde{C} C_{0}^{p}}{n-2} \frac{\log |x|}{|x|^{n-2}}
$$

$$
\Delta u_{2}+O\left(|x|^{-n-1}\right)+O\left(|x|^{2-2 n} \log |x|\right)=0
$$

near $x=\infty$. Since $u_{2}(x) \rightarrow 0$ as $x \rightarrow \infty$ and $2 n-2 \geqq n+1$, we conclude from the proof of Theorem 2.4 that $|x|^{n-2} u_{2}(x) \rightarrow c_{1}$ as $x \rightarrow \infty$, and that near $\infty$

$$
u_{2}(x)-\frac{c_{1}}{|x|^{n-2}}=O\left(\frac{1}{|x|^{n-1-\varepsilon}}\right)
$$

for any $\varepsilon>0$. (Note that $c_{1}$ may not be positive, but the proof of Theorem 2.4 still works.) Now we can proceed as in the previous case. Setting

$$
y=\frac{x}{|x|^{2}}, \quad v(x)=|x|^{2-n} u_{2}(y)=|y|^{n-2} u_{2}(y)=c_{1}+v_{1}(x)
$$

where $v_{1}(x)=O\left(|x|^{1-\varepsilon}\right)$ near $x=0$, we have, from (2.46), that near $x=0$

$$
\begin{aligned}
0= & \Delta v_{1}-\frac{\tilde{C} C_{0}^{p}}{|x|^{2}}+\frac{1}{|x|^{2}}\left[\tilde{C}+c|x|+c|x|^{2}+O\left(|x|^{\mu}\right)\right] \\
& \times\left[C_{0}-C|x|^{n-2} \log |x|+c_{1}|x|^{n-2}+|x|^{n-2} v_{1}(x)\right]^{p} .
\end{aligned}
$$

That is,

$$
\begin{aligned}
0= & \Delta v-\frac{\tilde{C} C_{0}^{p}}{|x|^{2}}+\frac{\tilde{C} C_{0}^{p}}{|x|^{2}}\left[1+c|x|+c|x|^{2}+O\left(|x|^{\mu}\right)\right] \\
& \times\left[1-C|x|^{n-2} \log |x|+c|x|^{n-2}+|x|^{n-2} v_{1}(x)+O\left(|x|^{2 n-4}(\log |x|)^{2}\right)\right]
\end{aligned}
$$


near $x=0$. Straightforward computation shows that

$0=\Delta v_{1}+\left[-C|x|^{n-4} \log |x|+c|x|^{n-4}+c|x|^{-1}+c+O\left(|x|^{n-3-\varepsilon}\right)+O\left(|x|^{\mu-2}\right)\right]$

near $x=0$. Since

$$
\Delta\left(|x|^{b+2} \log |x|\right)=(b+2)(b+n)|x|^{b} \log |x|+(n+2 b+2)|x|^{b},
$$

we derive

$$
0=\Delta\left(v_{1}-C|x|^{n-2} \log |x|\right)+c|x|^{n-4}+c|x|^{-1}+c+O\left(|x|^{n-3-\varepsilon}\right)+O\left(|x|^{\mu-2}\right)
$$

near $x=0$ for any $\varepsilon>0$. Now we need to consider two subcases separately: $n \geqq 4$ and $n=3$.

If $n \geqq 4$, (2.49) yields that

$$
v_{1}(x)=C|x|^{n-2} \log |x|+c|x|+a \cdot x+v_{2}(x)
$$

where $v_{2} \in C^{2}$ near $x=0$ with $v_{2}(0)=0, \nabla v_{2}(0)=0$ by similar arguments used in $\S 2$. As before, $v_{2}(x)=R(x)|x|$ near $x=0$ for some Lipschitz continuous function $R$ with $R(0)=0$. This establishes the first half of the following theorem.

Theorem 2.50. Let $u$ be a bounded positive solution of (2.31). Suppose that for some constants $\tilde{C}>0, \mu>2$

$$
K(x)=\frac{1}{|x|^{n}}\left(\tilde{C}+\frac{c}{|x|}+\frac{c}{|x|^{2}}+O\left(\frac{1}{|x|^{\mu}}\right)\right)
$$

near $x=\infty$. Then $C_{0}=\lim _{x \rightarrow \infty} u(x)$ always exists. If $C_{0}>0$, then for $n \geqq 4$,

$$
\begin{aligned}
u(x)= & C_{0}+\frac{\tilde{C} C_{0}^{p}}{n-2} \frac{\log |x|}{|x|^{n-2}}+\frac{c_{1}}{|x|^{n-2}}+\frac{c}{|x|^{n-1}}+\frac{a \cdot x}{|x|^{n}} \\
& -C \frac{\log |x|}{|x|^{2 n-4}}+R\left(\frac{x}{|x|^{2}}\right) \frac{1}{|x|^{n-1}}
\end{aligned}
$$

near $x=\infty$, and for $n=3$,

$$
\begin{aligned}
u(x)= & C_{0}+\tilde{C} C_{0}^{p} \frac{\log |x|}{|x|}+\frac{c_{1}}{|x|}-C \frac{\log |x|}{|x|^{2}}+\frac{c}{|x|^{2}}+\frac{a \cdot x}{|x|^{3}} \\
& +c \frac{(\log |x|)^{2}}{|x|^{3}}+c \frac{\log |x|}{|x|^{3}}+R\left(\frac{x}{|x|^{2}}\right) \frac{1}{|x|^{2}}
\end{aligned}
$$

near $x=\infty$, where $a \in \boldsymbol{R}^{n}$ is a constant vector and $R$ is a Lipschitz continuous function near $x=0$ with $R(0)=0$.

Completion of the Proof. If $n=3$, (2.49) becomes

$$
0=\Delta\left(v_{1}-C|x| \log |x|\right)+c|x|^{-1}+c+O\left(|x|^{-\varepsilon}\right)+O\left(|x|^{\mu-2}\right)
$$


near $x=0$. Since $\varepsilon>0$ can be arbitrarily small, we have, as in (2.21), that near $x=0$

$$
v_{1}(x)=C|x| \log |x|+c|x|+a \cdot x+v_{2}(x)
$$

where $v_{2} \in C^{1, \delta}$ near $x=0$ with $v_{2}(0)=0, \nabla v_{2}(0)=0$, for any $\delta<1$, i.e., $v_{2}(x)=O\left(|x|^{1+\delta}\right)$ near $x=0$ for any $\delta<1$. Substituting (2.53) back into (2.47), we obtain, after a somewhat tedious computation, that

$$
\begin{aligned}
0= & \Delta v_{1}-C|x|^{-1} \log |x|+c|x|^{-1}+C(\log |x|)^{2}+c \log |x| \\
& +c+C(a \cdot x)|x|^{-1}+O\left(|x|^{e}\right)
\end{aligned}
$$

near $x=0$. From (2.48) and the fact that

$$
\triangle\left(|x|^{2}(\log |x|)^{2}\right)=6(\log |x|)^{2}+10 \log |x|+2
$$

in $\boldsymbol{R}^{3}$, we may rewrite (2.54) as

$$
\begin{aligned}
0= & \Delta\left[v_{1}-C|x| \log |x|+C|x|^{2}(\log |x|)^{2}+c|x|^{2} \log |x|\right] \\
& +C(a \cdot x)|x|^{-1}+c|x|^{-1}+c+O\left(|x|^{\varepsilon}\right) .
\end{aligned}
$$

Thus it follows that near $x=0$,

$$
\begin{aligned}
v_{1}(x)= & C|x| \log |x|+c|x|^{2}(\log |x|)^{2}+c|x|^{2} \log |x| \\
& +C(a \cdot x)|x|+c|x|+\tilde{a} \cdot x+v_{3}(x)
\end{aligned}
$$

where $v_{3} \in C^{2, \varepsilon}$ near $x=0$ and $v_{3}(0)=0, \nabla v_{3}(0)=0$. Comparing (2.55) to (2.53) we see that $a=\tilde{a}$. Since we can rewrite $C(a \cdot x)|x|+v_{3}(x)$ as $R(x)|x|$, (2.52) follows immediately from (2.55). This finishes the proof of Theorem 2.50 .

Finally we turn to case $2<\tau<n$. Assuming that

$$
K(x)=|x|^{-\tau}\left(\tilde{C}+O\left(|x|^{-\mu}\right)\right)
$$

near $\infty$ where $\mu>\max \{0,1-\eta\}$ and $\eta=\tau-2$, we see that a bounded positive solution $u$ must satisfy (2.34), i.e., $u=C_{0}+u_{1}$ where $u_{1}$ is defined in (2.34); and by Lemma 2.3 in [LN1] we have

$$
0 \leqq u_{1}(x) \leqq C|x|^{2-\tau}
$$

near $x=\infty$. As in the previous case that $\tau=n$, we cannot apply the Kelvin transform directly to derive the asymptotic behavior of $u$; more care is needed. In fact, this case is harder than previous ones, and our estimates are not quite as explicit as before.

Let $k$ be the positive integer such that $k \eta<n-2 \leqq(k+1) \eta$. Then we set

$$
N_{l}(x)=\frac{1}{n(n-2) \omega_{n}} \int_{\mathbb{R}^{n}} \frac{\left(C_{0}+N_{l-1}(y)\right)^{p} K(y)}{|x-y|^{n-2}} d y, \quad l=1,2, \ldots, k+1,
$$


and $N_{0}(x) \equiv 0$. It follows again from Lemma 2.3 in [LN1] that

$$
0 \leqq N_{l}(x) \leqq C(1+|x|)^{-\eta}, \quad l=1,2, \ldots, k+1 .
$$

Now we assert that for $x \in \boldsymbol{R}^{n}$,

$$
\begin{gathered}
\left|N_{l}(x)-N_{l-1}(x)\right| \leqq C(1+|x|)^{-l \eta}, \quad l=1, \ldots, k, \\
\left|N_{k+1}(x)-N_{k}(x)\right| \leqq C(1+|x|)^{2-n} \log (|x|+2) .
\end{gathered}
$$

First, when $l=1,(2.60)$ follows from (2.59). For $1<l \leqq k$, we proceed by induction. By the induction hypothesis we have

$$
\begin{aligned}
\left|N_{l}(x)-N_{l-1}(x)\right| & \leqq C \int_{\boldsymbol{R}^{n}} \frac{\left|N_{l-1}(y)-N_{l-2}(y)\right| K(y)}{|x-y|^{n-2}} d y \\
& \leqq C \int_{\boldsymbol{R}^{n}} \frac{1}{(1+|y|)^{(l-1) \eta+\tau}} \cdot \frac{1}{|x-y|^{n-2}} d y \\
& =C \int_{|x|}^{\infty} \frac{1}{t^{n-1}} \int_{0}^{t} \frac{1}{(1+s)^{(l-1) \eta+\tau}} s^{n-1} d s d t \\
& \leqq \frac{C}{(1+|x|)^{l n}},
\end{aligned}
$$

for all $x \in \boldsymbol{R}^{n}$, and (2.60) is established. For (2.61) we have similarly that

$$
\begin{aligned}
\left|N_{k+1}(x)-N_{k}(x)\right| & \leqq C \int_{|x|}^{\infty} \frac{1}{t^{n-1}} \int_{0}^{t} \frac{1}{(1+s)^{k n+\tau}} s^{n-1} d s d t \\
& \leqq C \int_{|x|}^{\infty} \frac{1}{t^{n-1}} \int_{0}^{t} \frac{s^{n-1}}{1+s^{n}} d s d t \\
& \leqq C|x|^{2-n} \log |x|
\end{aligned}
$$

for $|x|$ large. Thus (2.61) holds.

Next we observe that

$$
u(x)=C_{0}+N_{l}(x)+u_{l+1}(x), \quad l=0,1, \ldots, k
$$

where for $l \geqq 1$,

$$
u_{l+1}(x)=\frac{1}{n(n-2) \omega_{n}} \int_{\boldsymbol{R}^{n}} \frac{\left[\left(C_{0}+N_{l-1}(y)+u_{l}(y)\right)^{p}-\left(C_{0}+N_{l-1}(y)\right)^{p}\right] K(y)}{|x-y|^{n-2}} d y .
$$


By (2.57) and the arguments used in deriving (2.60) and (2.61) we obtain

$$
0 \leqq u_{l+1}(x) \leqq C|x|^{-(l+1) \eta}, \quad \text { for } l=0,1, \ldots, k-1
$$

near $x=\infty$, and

$$
0 \leqq u_{k+1}(x) \leqq C|x|^{2-n} \log |x|
$$

near $x=\infty$. Setting $l=k$ in (2.62) and iterating one step further, we have

$$
\Delta u+K\left(C_{0}+N_{k}\right)^{p}+K\left[\left(C_{0}+N_{k}+u_{k+1}\right)^{p}-\left(C_{0}+N_{k}\right)^{p}\right]=0 .
$$

Hence

$$
u(x)=C_{0}+N_{k+1}(x)+u_{k+2}(x)
$$

where $u_{k+2}$ is given by (2.63) with $l=k+1$. Since

$$
\left[\left(C_{0}+N_{k}(y)+u_{k+1}(y)\right)^{p}-\left(C_{0}+N_{k}(y)\right)^{p}\right] K(y)=O\left(|y|^{-n-\eta} \log |y|\right)
$$

near $y=\infty$, we conclude from the proof of Theorem 2.4 that

$$
\begin{aligned}
\lim _{x \rightarrow \infty}|x|^{n-2} u_{k+2}(x) & =\frac{1}{n(n-2) \omega_{n}} \int_{R^{n}}\left[\left(C_{0}+N_{k}+u_{k+1}\right)^{p}-\left(C_{0}+N_{k}\right)^{p}\right] K d y \\
& \equiv C_{k+2}
\end{aligned}
$$

and

$$
u_{k+2}(x)-\frac{C_{k+2}}{|x|^{n-2}}=O\left(\frac{1}{|x|^{\delta+n-2}}\right)
$$

near $x=\infty$ for all $\delta<\min \{1, \eta\}$.

We are now ready to apply the Kelvin transform. We set

$$
y=\frac{x}{|x|^{2}}, \quad v(x)=|x|^{2-n} u_{k+2}(y) \equiv C_{k+2}+v_{1}(x)
$$

where $v_{1}$ is defined by the last equality and therefore $v_{1}(x)=O\left(|x|^{\delta}\right)$ near $x=0$ for all $\delta<\min \{1, \eta\}$. Straightforward computation shows that

$$
\begin{aligned}
0= & \Delta v+|x|^{-n-2} K\left(\frac{x}{|x|^{2}}\right) \\
& \times\left\{\left[C_{0}+N_{k+1}\left(\frac{x}{|x|^{2}}\right)+C_{k+2}|x|^{n-2}+|x|^{n-2} v_{1}(x)\right]^{p}-\left[C_{0}+N_{k}\left(\frac{x}{|x|^{2}}\right)\right]^{p}\right\} .
\end{aligned}
$$

Thus we have

$$
\begin{aligned}
0= & \Delta v+|x|^{-n-2} K\left(\frac{x}{|x|^{2}}\right) \\
& \times\left\{\left[C_{0}+N_{k+1}\left(\frac{x}{|x|^{2}}\right)+C_{k+2}|x|^{n-2}\right]^{p}-\left[C_{0}+N_{k}\left(\frac{x}{|x|^{2}}\right)\right]^{p}\right\}+f_{1}(x)
\end{aligned}
$$


where $f_{1}$ is defined by (2.67) and the last equality, and $f_{1}(x)=O\left(|x|^{\eta+\delta-2}\right)$ near $x=0$ for any $\delta<\min \{1, \eta\}$ since $\varepsilon$ may be chosen so small that $2(n-2)-\varepsilon>n-2+\delta$. Using (2.56) and (2.61) we derive from the above formula that near $x=0$

$$
\begin{aligned}
0= & \Delta v+\tilde{C}|x|^{\eta-n}\left\{\left[C_{0}+N_{k+1}\left(\frac{x}{|x|^{2}}\right)+C_{k+2}|x|^{n-2}\right]^{p}-\left[C_{0}+N_{k}\left(\frac{x}{|x|^{2}}\right)\right]^{p}\right\} \\
& +f_{1}(x)+g_{1}(x),
\end{aligned}
$$

where $g_{1}$ is defined by (2.68) and $g_{1}(x)=O\left(|x|^{\eta+\mu-2-\varepsilon}\right)$ near $x=0$ for any $\varepsilon>0$ arbitrarily small. Note that $\eta+\mu-2-\varepsilon>-1$ if $\varepsilon$ is sufficiently small and, near $x=0$, that

$$
\begin{aligned}
{\left[C_{0}+N_{k+1}\left(\frac{x}{|x|^{2}}\right)+C_{k+2}|x|^{n-2}\right]^{p} } & -\left[C_{0}+N_{k}\left(\frac{x}{|x|^{2}}\right)\right]^{p} \\
& =O\left(\left|N_{k+1}-N_{k}\right|\left(\frac{x}{|x|^{2}}\right)+C_{k+2}|x|^{n-2}\right) .
\end{aligned}
$$

To continue, we first introduce the following notation. Let $f$ be a continuous function in a deleted neighborhood of the origin, say, $B_{1}(0) \backslash\{0\}$, with $f(x)=O\left(|x|^{\sigma}\right)$ at $x=0$ for some $\sigma>-2$. Set

$$
W(f)(x)=\frac{1}{n(n-2) \omega_{n}}\left(\int_{\boldsymbol{R}^{n}} \frac{f(y)}{|x-y|^{n-2}} d y-\int_{\boldsymbol{R}^{n}} \frac{f(y)}{|y|^{n-2}} d y\right)
$$

where $f$ is defined to be identically zero outside $B_{1}(0)$ for convenience. Note that $W(f)(0)=0$ and $W(f)(x)=O\left(|x|^{\sigma^{\prime}}\right)$ at $x=0$ for any $\sigma^{\prime}<2+\sigma$ and $\sigma^{\prime} \leqq 1$.

Then it follows from (2.68) and the argument leading to (2.21) and (2.22) that near $x=0$

$$
v(x)= \begin{cases}C_{k+2}+W_{1}(x)+v_{2}(x) & \text { if } 2 \eta \leqq 1, \\ C_{k+2}+W_{1}(x)+a \cdot x+\tilde{v}_{2}(x) & \text { if } 2 \eta>1,\end{cases}
$$

where $v_{2}(x)=O\left(|x|^{\eta+\delta}\right)$ near $x=0$ for all $\delta<\eta, \tilde{v}_{2}(x)=O\left(|x|^{\rho}\right)$ at $x=0$ for any $\rho<\min \{2 \eta, \eta+\mu, 2\}, a$ is a constant vector $\boldsymbol{R}^{n}$ and

$$
W_{1}(x)=W\left(\tilde{C}|x|^{n-n}\left\{\left[C_{0}+N_{k+1}\left(\frac{x}{|x|^{2}}\right)+C_{k+2}|x|^{n-2}\right]^{p}-\left[C_{0}+N_{k}\left(\frac{x}{|x|^{2}}\right)\right]^{p}\right\}\right) \text {. }
$$

Observe that $\tilde{v}_{2} \in C^{1}$ at $x=0$.

In case $2 \eta \leqq 1$, we proceed as follows. Substituting (2.70) into (2.67) we obtain, as in (2.68), that

$$
\begin{aligned}
& 0=\Delta v \\
& +\tilde{C}|x|^{\eta-n}\left\{\left[C_{0}+N_{k+1}\left(\frac{x}{|x|^{2}}\right)+C_{k+2}|x|^{n-2}+|x|^{n-2} W_{1}(x)\right]^{p}-\left[C_{0}+N_{k}\left(\frac{x}{|x|^{2}}\right)\right]^{p}\right\} \\
& +O\left(|x|^{2 \eta+\delta-2}\right)+O\left(|x|^{\eta+\mu-2-\varepsilon}\right)
\end{aligned}
$$


near $x=0$ for any $\delta<\eta$ and any $\varepsilon>0$ small. As before we conclude that near $x=0$

$$
v(x)= \begin{cases}C_{k+2}+W_{2}(x)+v_{3}(x) & \text { if } 3 \eta \leqq 1, \\ C_{k+2}+W_{2}(x)+a \cdot x+\tilde{v}_{3}(x) & \text { if } 3 \eta>1,\end{cases}
$$

where $v_{3}(x)=O\left(|x|^{2 \eta+\delta}\right)$ at $x=0, \quad \tilde{v}_{3}(x)=O\left(|x|^{\rho}\right)$ at $x=0$ for any $\rho<\min \{3 \eta, \eta+\mu\}$ (thus $\tilde{v}_{3} \in C^{1}$ near $x=0$ ), $a$ is a constant vector in $\boldsymbol{R}^{n}$ and

$$
\begin{aligned}
W_{2}(x)=W\left(\tilde{C}|x|^{\eta-n}\{\right. & {\left[C_{0}+N_{k+1}\left(\frac{x}{|x|^{2}}\right)+C_{k+2}|x|^{n-2}+|x|^{n-2} W_{1}(x)\right]^{p} } \\
& \left.\left.-\left[C_{0}+N_{k}\left(\frac{x}{|x|^{2}}\right)\right]^{p}\right\}\right) .
\end{aligned}
$$

Iterating this process we finally arrive at

$$
v(x)=C_{k+2}+W_{h}(x)+a \cdot x+\tilde{v}_{h+1}(x)
$$

near $x=0$, where $a$ is a constant vector in $\boldsymbol{R}^{n}, h$ is the first positive integer such that $(h+1) \eta>1, \tilde{v}_{h+1}(x)=O\left(|x|^{\rho}\right)$ at $x=0$ for any $\rho<$ $\min \{(h+1) \eta, \eta+\mu, 2\}$ and $W_{l}$ is defined recursively by $W_{0} \equiv 0$ and

$$
\begin{aligned}
W_{l+1}(x)=W\left(\tilde{C}|x|^{\eta-n}\{\right. & {\left[C_{0}+N_{k+1}\left(\frac{x}{|x|^{2}}\right)+C_{k+2}|x|^{n-2}+|x|^{n-2} W_{l}(x)\right]^{p} } \\
& \left.\left.-\left[C_{0}+N_{k}\left(\frac{x}{|x|^{2}}\right)\right]^{p}\right\}\right)
\end{aligned}
$$

near $x=0$. Since $\tilde{v}_{h+1} \in C^{1}$ near $x=0$, it follows that

$$
v(x)=C_{k+2}+W_{h}(x)+R(x)
$$

where $R$ is in $C^{1}$ (thus Lipschitz continuous) near $x=0$. This together with (2.64) and (2.66) imply the following

Theorem 2.75. Let $u$ be a bounded positive solution of (2.31). Suppose that $K \geqq 0$ in $\boldsymbol{R}^{n}, n \geqq 3$ and that

$$
K(x)=|x|^{-\tau}\left(\tilde{C}+O\left(|x|^{-\mu}\right)\right)
$$

near $x=\infty$ for some constants $2<\tau<n, \tilde{C}>0$ and $\mu>\max \{0,1-\eta\}$ where $\eta=\tau-2$. Then $C_{0}=\lim _{x \rightarrow \infty} u(x)$ always exists. If $C_{0}>0$, then

$$
u(x)=C_{0}+N_{k+1}(x)+\frac{C_{k+2}}{|x|^{n-2}}+\frac{1}{|x|^{n-2}} W_{h}\left(\frac{x}{|x|^{2}}\right)+R\left(\frac{x}{|x|^{2}}\right) \frac{1}{|x|^{n-2}}
$$

near $x=\infty$, where $k$ is the integer such that $k \eta<n-2 \leqq(k+1) \eta$, $h$ is the first positive integer such that $(h+1) \eta>1$, and $N_{k+1}$ is defined by $(2.58)$. $W_{h}$ is given by (2.73) and (2.69), the constant $C_{k+2}$ is defined in (2.65) and $R$ is a Lipschitz continuous function near 0 with $R(0)=0$. 


\subsection{Further Extensions}

The methods we used in Subsections 2.1 and 2.2 can also be applied to the study of the asymptotic behavior of solutions for more general equations and for domains other than the entire $\boldsymbol{R}^{n}$. In this subsection we shall indicate some such generalizations.

First, we consider domains other than $\boldsymbol{R}^{n}$, and we have

Remark 2.77. Theorems $2.8,2.12,2.16,2.41,2.50$ and 2.75 also hold for the corresponding exterior domain problems with similar proofs.

Next, we discuss more detailed higher-order expansions of solutions of (2.1). Although it suffices for our later applications to expand the solutions of equation (2.1) up to the order of $|x|^{-n}$ near $x=0$ (such as in (2.11), (2.15), (2.17), (2.42), (2.43), (2.44), (2.51) and (2.52)) or up to the order of $|x|^{-(n-1)}$ (such as in (2.76)), we could, by continuing the iteration process there, obtain expansions up to any order provided that $K$ also has a similar higher-order expansion. For instance, in Theorem 2.75 once we have (2.76), we then set

$$
u_{k+3}(x)=u(x)-\left[C_{0}+N_{k+1}(x)+\frac{C_{k+2}}{|x|^{n-2}}+\frac{1}{|x|^{n-2}} W_{h}\left(\frac{x}{|x|^{2}}\right)\right]
$$

and let $v_{k+3}$ be the inversion of $u_{k+3}$ given by the Kelvin transform (2.66). Now in $B_{1}(0) \backslash\{0\}, v_{k+3}$ satisfies

$$
\begin{aligned}
& 0= \Delta v_{k+3}(x)+\frac{1}{|x|^{n+2}} K\left(\frac{x}{|x|^{2}}\right)\left\{\left[C_{0}+N_{k+1}\left(\frac{x}{|x|^{2}}\right)+C_{k+2}|x|^{n-2}\right.\right. \\
&\left.\left.+|x|^{n-2} W_{h}(x)+|x|^{n-2} v_{k+3}(x)\right]^{p}-\left[C_{0}+N_{k}\left(\frac{x}{|x|^{2}}\right)\right]^{p}\right\} \\
&-\tilde{C}|x|^{n-n}\left\{\left[C_{0}+N_{k+1}\left(\frac{x}{|x|^{2}}\right)+C_{k+2}|x|^{n-2}+|x|^{n-2} W_{h-1}(x)\right]^{p}\right. \\
&\left.-\left[C_{0}+N_{k}\left(\frac{x}{|x|^{2}}\right)\right]^{p}\right\} .
\end{aligned}
$$

Hence we have as in (2.68) that

$$
\begin{aligned}
0= & \Delta v_{k+3}(x)+\tilde{C}|x|^{\eta-n}\left\{\left[C_{0}+N_{k+1}\left(\frac{x}{|x|^{2}}\right)+C_{k+2}|x|^{n-2}+|x|^{n-2} W_{h}(x)\right]^{p}\right. \\
& \left.-\left[C_{0}+N_{k+1}\left(\frac{x}{|x|^{2}}\right)+C_{k+2}|x|^{n-2}+|x|^{n-2} W_{h-1}(x)\right]^{p}\right\}+f_{2}(x) \\
= & \Delta v_{k+3}(x)+g_{2}(x)+f_{2}(x),
\end{aligned}
$$


where $f_{2}$ and $g_{2}$ are defined by (2.79), and it is easy to check that

$$
\begin{aligned}
& f_{2}(x)=O\left(|x|^{\eta-1}+|x|^{\mu+\eta-2} \log \frac{1}{|x|}\right), \\
& g_{2}(x)=g_{2}(|x|)=O\left(|x|^{(n+1) \eta-2} \log \frac{1}{|x|}\right)
\end{aligned}
$$

near $x=0$.

From (2.79) and (2.80) we have

$$
v_{k+3}(x)=W\left(g_{2}\right)(|x|)+\tilde{v}_{k+4}(x)
$$

where $\tilde{v}_{k+4} \in C^{1, \delta}\left(B_{1}(0)\right)$ for any $\delta \leqq \eta$ and $\delta<\mu+\eta-1$ with $\tilde{v}_{k+4}(0)=0$. In particular, it follows that near $x=0$,

$$
v_{k+3}(x)=W\left(g_{2}\right)(|x|)+b \cdot x+v_{k+4}(x)
$$

where $b$ is a constant vector in $\boldsymbol{R}^{n}$ and $v_{k+4}(x)=O\left(|x|^{\min (\delta+1,2)}\right)$ near $x=0$. Therefore if it is assumed that $\mu+\eta>2, \eta>1$, then by the same iteration process which was used in deriving (2.74) we have

$$
v_{k+3}(x)=W\left(g_{2}\right)(|x|)+b \cdot x+\tilde{W}(x)+|x| R(x)
$$

near $x=0$ where $\tilde{W}$ is the Newtonian potential of a function of $W\left(g_{2}\right)$, and $R(x)$ is Lipschitz continuous with $R(0)=0$. Thus

$$
\begin{aligned}
u(x)= & C_{0}+N_{k+1}(x)+\frac{C_{k+2}}{|x|^{n-2}}+\frac{1}{|x|^{n-2}} W_{h}\left(\frac{x}{|x|^{2}}\right) \\
& +\frac{b \cdot x}{|x|^{n}}+\frac{1}{|x|^{n-2}} \tilde{W}\left(\frac{x}{|x|^{2}}\right)+\frac{1}{|x|^{n-1}} R\left(\frac{x}{|x|^{2}}\right)
\end{aligned}
$$

near $x=\infty$.

Finally we consider equations more general than (2.31). In Subsection 2.2 the simple equation (2.31) was studied because more precise asymptotic expansions, namely, (2.42), (2.43) and (2.51), can be obtained. However, our method does apply to more general equations. To illustrate this, we list below the corresponding results for the equation

$$
\Delta u+K(x) f(u)=0 \quad \text { in } \boldsymbol{R}^{n}, n \geqq 3,
$$

where $K \geqq 0, f(0)=0$ and $f \in C^{1}(0, \infty) \cap C^{\alpha}[0, \infty)$ for some $0 \leqq \alpha<1$.

Theorem 2.83. Let $u$ be a bounded positive solution of (2.82). If $K(x)=O\left(|x|^{-\tau}\right)$ near $x=\infty$ for some $\tau>2$, then $C_{0}=\lim _{x \rightarrow \infty} u(x)$ always exists. Moreover, if $C_{0}=0$, then $u(x)=O\left(|x|^{2-n}\right)$ near $\infty$ provided that $\alpha>\frac{n-\tau}{n-2}$. If $C_{0}>0$ and $K \sim|x|^{-\tau}$ near $\infty$, then

$$
\left(u(x)-C_{0}\right) \sim \begin{cases}C|x|^{2-n} & \text { when } \tau>n, \\ C|x|^{2-n} \log |x| & \text { when } \tau=n, \\ C|x|^{2-\tau} & \text { when } 2<\tau<n .\end{cases}
$$


The proof is similar to that of Theorem 2.32 , which may be found in [LN1].

For the case that $C_{0}>0$, the next three theorems are the counterparts of Theorems 2.41, 2.50 and 2.75 .

Theorem 2.84. Let $u$ be a bounded positive solution of (2.82). Suppose that $K(x)=$ $O\left(|x|^{-\tau}\right)$ near $\infty$ for some $\tau>n$ and that $\gamma=\tau-n$. Then $C_{0}=\lim _{x \rightarrow \infty} u(x)$ always exists. If $C_{0}>0$, then the following statements hold.

(i) If $\tau>n+1$ and $K(x)=|x|^{-\tau}\left(\tilde{C}+O\left(|x|^{-\mu}\right)\right)$ near $\infty$ for some $\mu>$ $\max \{0,2-\gamma\}$, then near $\infty$,

$$
u(x)=C_{0}+\frac{C_{1}}{|x|^{n-2}}+\frac{1}{|x|^{n-2}} W_{1}^{*}\left(\frac{1}{|x|}\right)+\frac{a \cdot x}{|x|^{n}}+\frac{1}{|x|^{n-1}} R\left(\frac{x}{|x|^{2}}\right) .
$$

(ii) If $\tau \leqq n+1$ and $K(x)=|x|^{-\tau}\left(\tilde{C}+\tilde{c}|x|^{-1}+O\left(|x|^{-\mu}\right)\right)$ near $\infty$ for some $\mu>2-\gamma$, then

$$
u(x)=C_{0}+\frac{C_{1}}{|x|^{n-2}}+\frac{1}{|x|^{n-2}} W_{2}^{*}\left(\frac{1}{|x|}\right)+\frac{a \cdot x}{|x|^{n}}+\frac{1}{|x|^{n-1}} R\left(\frac{x}{|x|^{2}}\right)
$$

near $x=\infty$ when $\tau=n+1$,

$$
u(x)=C_{0}+\frac{C_{1}}{|x|^{n-2}}+\frac{1}{|x|^{n-2}} W_{3}^{*}\left(\frac{1}{|x|}\right)+\frac{a \cdot x}{|x|^{n}}+\frac{1}{|x|^{n-1}} R\left(\frac{x}{|x|^{2}}\right)
$$

near $x=\infty$ when $\tau<n+1$, where

$$
\begin{aligned}
& W_{1}^{*}(|x|)=W\left(\tilde{C}|x|^{\gamma-2} f\left(C_{0}+C_{1}|x|^{n-2}\right)\right), \\
& W_{2}^{*}(|x|)=W\left(|x|^{\gamma-2}(\tilde{C}+\tilde{c}|x|) f\left(C_{0}+C_{1}|x|^{n-2}\right)\right), \\
& W_{3}^{*}(|x|)=W\left(|x|^{\gamma-2}(\tilde{C}+\tilde{c}|x|) f\left(C_{0}+|x|^{n-2}\left(C_{1}+W_{2}^{*}(|x|)\right)\right)\right)
\end{aligned}
$$

with $W$ given by (2.69).

Since the proof is similar to that of Theorem 2.41 , we shall be brief and only sketch a few key steps for the case that $n<\tau<n+1$. First, we write $u(x)=C_{0}+u_{1}(x)$. By the proof of Theorem 2.4, there exists a positive constant $C_{1}$ such that

$$
|x|^{n-2} u_{1}(x)-C_{1}=O\left(|x|^{-\gamma}\right)
$$

for $x$ near $\infty$. Then let $v=C_{1}+v_{1}$ be the inversion of $u_{1}$ defined in (2.36), and we have (similar to (2.38))

$$
\begin{aligned}
0 & =\Delta v+|x|^{\gamma-2}\left(\tilde{C}+\tilde{c}|x|+O\left(|x|^{\mu}\right)\right) f\left(C_{0}+|x|^{n-2}\left(C_{1}+v_{1}(x)\right)\right) \\
& =\Delta v+|x|^{\gamma-2}(\tilde{C}+\tilde{c}|x|) f\left(C_{0}+C_{1}|x|^{n-2}\right)+O\left(|x|^{\mu+\gamma-2}+|x|^{n+2 y-4}\right)
\end{aligned}
$$

near $x=0$. Therefore by arguments used in Subsection 2.2 we have

$$
v(x)=C_{1}+W_{2}^{*}(|x|)+v_{2}(x)
$$


where $v_{2}(0)=0$ and $v_{2} \in C^{1}\left(B_{1}(0)\right)$ since $\min (\mu+\gamma, n+2 \gamma-2)>1$. Now, substituting (2.89) back into (2.88), we obtain

$$
\begin{aligned}
0= & \Delta v+|x|^{\gamma-2}(\tilde{C}+\tilde{c}|x|) f\left(C_{0}+|x|^{n-2}\left(C_{1}+W_{2}^{*}(|x|)\right)\right) \\
& +O\left(|x|^{\mu+\gamma-2}+|x|^{n+\gamma-3}\right)
\end{aligned}
$$

near $x=0$. Similarly it follows that

$$
v(x)=C_{1}+W_{3}^{*}(|x|)+a \cdot x+|x| R(x)
$$

near $x=0$, where $a \in \boldsymbol{R}^{n}, R$ is Lipschitz continuous and $R(0)=0$, since $\min (\mu+\gamma, n+\gamma-1)>2$. Thus (2.87) is proved.

Theorem 2.90. Let $u$ be a bounded positive solution of (2.82). Suppose that for some constants $\tilde{C}>0, c_{1}, c_{2}$ and $\mu>2$

$$
K(x)=\frac{1}{|x|^{n}}\left(\tilde{C}+\frac{c_{1}}{|x|}+\frac{c_{2}}{|x|^{2}}+O\left(\frac{1}{|x|^{\mu}}\right)\right)
$$

near $x=\infty$. Then $C_{0}=\lim _{x \rightarrow \infty} u(x)$ always exists. If $C_{0}>0$, then, for $n \geqq 4$,

$u(x)=C_{0}+N_{f, 1}(x)+\frac{c}{|x|^{n-2}}+\frac{1}{|x|^{n-2}} W_{4}^{*}\left(\frac{x}{|x|^{2}}\right)+\frac{a \cdot x}{|x|^{n}}+\frac{1}{|x|^{n-1}} R\left(\frac{x}{|x|^{2}}\right)$

near $x=\infty$, and, for $n=3$,

$$
u(x)=C_{0}+N_{f, 1}(x)+\frac{c}{|x|}+\frac{1}{|x|} W_{5}^{*}\left(\frac{x}{|x|^{2}}\right)+\frac{a \cdot x}{|x|^{3}}+\frac{1}{|x|^{2}} R\left(\frac{x}{|x|^{2}}\right)
$$

near $x=\infty$, where

$$
N_{f, l}(x) \frac{1}{n(n-2) \omega_{n}} \int_{R^{n}} \frac{f\left(C_{0}+N_{f, l-1}(y)\right) K(y)}{|x-y|^{n-2}} d y, \quad l=1,2, \ldots
$$

with $N_{f, 0} \equiv 0$, and

$$
\begin{aligned}
& W_{4}^{*}(x)= W\left(|x|^{2}\left(\tilde{C}+c_{1}|x|+c_{2}|x|^{2}\right)\left[f\left(C_{0}+N_{f, 1}\left(\frac{x}{|x|^{2}}\right)+|x|^{n-2} C_{1}\right)-f\left(C_{0}\right)\right]\right), \\
& W_{5}^{*}(x)=W\left(|x|^{-2}\left(\tilde{C}+c_{1}|x|+c_{2}|x|^{2}\right)\right. \\
&\left.\times\left[f\left(C_{0}+N_{f, 1}\left(\frac{x}{|x|^{2}}\right)+|x|\left(C_{1}+W_{4}^{*}(x)\right)\right)-f\left(C_{0}\right)\right]\right)
\end{aligned}
$$

near $x=0$.

Theorem 2.94. Let $u$ be $a$ bounded positive solution of (2.82). Suppose that $K(x)=|x|^{-\tau}\left(\tilde{C}+O\left(|x|^{-\mu}\right)\right)$ near $x=\infty$ for some constants $2<\tau<n, \tilde{C}>0$ and $\mu>\max \{0,1-\eta\}$ with $\eta=\tau-2$. Then $C_{0}=\lim _{x \rightarrow \infty} u(x)$ always exists. 
If $C_{0}>0$, then

$$
u(x)=C_{0}+N_{f, k+1}(x)+\frac{c_{k+2}}{|x|^{n-2}}+\frac{1}{|x|^{n-2}} \tilde{W}_{h}\left(\frac{x}{|x|^{2}}\right)+\frac{1}{|x|^{n-2}} R\left(\frac{x}{|x|^{2}}\right)
$$

near $x=\infty$, where $k$ is the first integer such that $k \eta<n-2 \leqq(k+1) \eta, h$ is the first positive integer such that $(h+1) \eta>1, N_{f, k+1}$ is defined by $(2.93), R$ is Lipschitz continuous near 0 with $R(0)=0$, and

$$
\begin{gathered}
\tilde{W}_{l+1}(x)=W\left(\tilde { C } | x | ^ { \eta - n } \left[f \left(C_{0}+N_{f, k+1}\left(\frac{x}{|x|^{2}}\right)+|x|^{n-2}\left(c_{k+2}+\tilde{W}_{l}(x)\right)\right.\right.\right. \\
\left.\left.-f\left(C_{0}+N_{f, k}\left(\frac{x}{|x|^{2}}\right)\right)\right]\right)
\end{gathered}
$$

$l=0,1,2, \ldots$ with $\tilde{W}_{0} \equiv 0$.

The proofs of Theorems 2.90 and 2.94 are omitted here since they are similar to those of Theorems 2.50 and 2.75, respectively. (We should perhaps point out that the constant $c_{k+2}$ in (2.95) and (2.96) is given by

$$
c_{k+2}=\frac{1}{n(n-2) \omega_{n}} \int_{R^{n}} K\left[f\left(C_{0}+N_{f, k}+u_{k+1}\right)-f\left(C_{0}+N_{f, k}\right)\right]
$$

which may or may not be positive. Similarly, the $u_{l}$ 's appearing in (2.62) may also change sign. Nevertheless, the same arguments go through with only minor modifications.)

Acknowledgment. This research was supported in part by the National Science Foundation.

\section{References}

[GNN] B. GIDAS, W.-M. Ni \& L. NIRENBERG, Symmetry of positive solutions of nonlinear elliptic equations in $\boldsymbol{R}^{n}$, Advances in Math. Supplementary Studies 7 A (L. NAChBin ed.) (1981), 369-402.

[H] H. Hopf, Differential Geometry in the Large, Lecture Notes in Mathematics Vol. 1000, Springer-Verlag 1983.

[LN1] Y. LI \& W.-M. NI, On conformal scalar curvature equations in $\boldsymbol{R}^{n}$, Duke Math J. 57 (1988), 895-924.

[LN2] Y. LI \& W.-M. NI, On the existence and symmetry properties of finite total mass solutions of the Matukuma equation, the Eddington equation and their generalizations, Arch. Rational Mech. Anal. 108 (1989), 175-194.

[Na] M. NaITo, A note on bounded positive entire solutions of semilinear elliptic equations, Hiroshima Math. J. 14 (1984), 211-214. 
[N] W.-M. Ni, On the elliptic equation $\Delta u+K(x) u^{\frac{n+2}{n-2}}=0$, its generalizations and applications in geometry, Indiana Univ. Math. J. 31 (1982), 493-529.

[NY] W.-M. Ni \& S. Yotsutani, Semilinear elliptic equations of Matukuma-type and related topics, Japan J. Appl. Math. 5 (1988), 1-32.

[S] J. SERRIN, A symmetry problem in potential theory, Arch. Rational Mech. Anal. 43 (1971), 304-318. 